\title{
Theory and Experiment on Sound Reduction by an Irregularly-Edged Screen over a Hard Ground
}

\author{
Chun Biao Gan ${ }^{1,2}$, Kai Ming $\mathrm{Li}^{1}$ and Hon Yiu Wong ${ }^{1}$ \\ ${ }^{1}$ Department of Mechanical Engineering, The Hong Kong Polytechnic University, Hong Kong, P.R. China \\ ${ }^{2}$ Department of Mechanics, CMEE, Zhejiang University, Hangzbou, 310027, P. R. China
}

(Received 1 July 2003; accepted 20 October 2003)

\begin{abstract}
Diffraction by a thin barrier with a square-wave form, a triangle-wave form or a randomly jagged top edge is investigated. The top edge of the sound barrier is divided into many infinitesimal segments with different heights to act as a series of sound sources. Asymptotic approximations are developed for predicting the diffraction field on the hard ground in the shadow zone of these three types of barriers from the theory of Menounou's. ${ }^{26}$ Based on the theory, the excess attenuation vs. the diffracted field by the straight barrier with the maximum height is simulated. Laboratory measurements are reported for comparison with the excess attenuation of triangle-wave form and randomly jagged-edged barriers by the present approximations. Differences between the present approximations, current existing models and the experimental data are very small. It is shown that the barrier with the triangle-wave form or randomly jagged edge can perform better to reduce the diffraction field over a wide frequency range even if the referenced sound field is taken as the diffracted field by the straight barrier with the maximum height. Moreover, by simple simulations, it is found that the randomly jagged barrier behaves just like a straight one when the source or the receiver is far away from the barrier, and almost the same excess attenuation can be observed as long as the horizontal width of each segment is made small enough for the barrier with a triangle-waveform edge or a randomly jagged edge. The formula presented here is very fast, simple and intuitive and this idea can be applied to the study of sound fields diffracted by many other types of irregular barriers.
\end{abstract}

\section{INTRODUCTION}

In recent years, noise pollution from highways, railroads, airports, large machinery and plants, etc., has become a major problem in modern urban life. The most common measure for noise reduction is to block the line-of-sight from the source to the receiver, so that only a very small portion of the noise can arrive at the listeners via diffraction over the barrier top or around its ends, via reflection from other buildings, and via scattering and refraction in the atmosphere.

The shape of the sound barriers can, in general, have shapes like thin screens, wedges or other kinds. ${ }^{1-5} \mathrm{Up}$ to the present, almost all the theoretical studies were focused on the diffraction field in the shadow zone of a barrier with a simple shape. These studies originated from Sommerfeld's exact solution for the diffraction of a plane wave by a semi-infinite plane screen or a wedge ${ }^{6}$ and from the Huygens-Fresnel principle, ${ }^{7}$ which is the basis for Kirchhoff's and various subsequent approximations of the diffracted field near the shadow boundary. Another theoretical method of deriving approximate solutions of complex diffraction problems was developed by Keller. ${ }^{8}$ His geometrical theory of diffraction can lead to relatively simple formulas, which combine the practicability of Kirchhoff's approximations with the greater accuracy of the Sommerfeld-type solutions and can be generalised to treat diffraction by three-dimensional objects of any smooth shape.

For a comparatively irregular barrier, it is rather difficult to perform the theoretical analysis on the diffraction field. Maekawa introduced an ingenious technique for replacing barriers of a more general shape by an equivalent thin screen, but there appears to be relatively little theoretical basis to this technique except in the case of small-angle diffraction by wedges. ${ }^{9}$ Therefore, some numerical methods are introduced to discuss the diffraction by solving the Helmholtz-Kirchhoff integral equation directly., ${ }^{5,10-15}$ Another alternative method is to deal with experimental investigations using scale models. ${ }^{9,16-21}$

In the beginning of the $1990 \mathrm{~s}$, Bailey et al. first introduced an improved measure to reduce the diffracted field by a barrier with random-edged profile. ${ }^{22}$ In their work, a jagged edge was used to affect the diffraction caused by circular apertures, disks, and rectangular barrier plates. Underwater measurements with very short pulses from a spark source showed that the scattered signal from an aperture (or disk) can practically disappear when the aperture (or disk) edge is made sufficiently jagged. Measurements with a rectangular barrier also showed degradation of the scattered signal when the barrier edge was made jagged.

Thereafter, Ho et al. ${ }^{23}$ studied the noise reduction by a barrier having a random-edged profile by a three-level, fullfactorial experiment and found that the acoustical performance of noise barriers can be significantly improved by introducing a random fluctuation in the height of the barrier edge. Menounou $^{24}$ analysed the RMS (root mean square) value of the edge wave on axis behind an aperture (or a disk) having a randomly jagged edge based on the Helmholtz-Kirchhoff integral theorem. She showed that the RMS value of the edge wave behind a noncircular aperture is less than that behind a circular aperture. She also showed that the difference increases as the aperture profile is made more randomly jagged, provided the delay time variations are not small compared to the characteristic period of the incident wave. Recently, Shao et al. ${ }^{25}$ simulated the insertion loss by noise barriers with random-edged profiles by the Huygens-Rayleigh integral, and suggested that one could make noise barriers more effective by creating random edges even if the average 